A N N A L E S Annales de Bretagne et des Pays de l'Ouest

\title{
Les doubles funérailles d'Anne de Bretagne. Le corps et le cœur (janvier-mars 1514)
}

\section{Michael Jones}

\section{(2) OpenEdition \\ 9 Journals}

\section{Édition électronique}

URL : http://journals.openedition.org/abpo/3745

DOI : $10.4000 /$ abpo.3745

ISBN : 978-2-7535-7411-3

ISSN : 2108-6443

\section{Éditeur}

Presses universitaires de Rennes

\section{Édition imprimée}

Date de publication : 20 décembre 2017

Pagination : 198-200

ISBN : 978-2-7535-7409-0

ISSN : 0399-0826

\section{Référence électronique}

Michael Jones, "Les doubles funérailles d'Anne de Bretagne. Le corps et le cœur (janvier-mars 1514) 》, Annales de Bretagne et des Pays de l'Ouest [En ligne], 124-4 | 2017, mis en ligne le 20 décembre 2019 consulté le 23 septembre 2020. URL : http://journals.openedition.org/abpo/3745 ; DOI : https:// doi.org/10.4000/abpo.3745

Ce document a été généré automatiquement le 23 septembre 2020

(c) Presses universitaires de Rennes 


\title{
Les doubles funérailles d'Anne de Bretagne. Le corps et le cœur (janvier-
} mars 1514)

\author{
Michael Jones
}

\section{RÉFÉRENCE}

SANTROT, Jacques, Les doubles funérailles d'Anne de Bretagne. Le corps et le cœur (janvier-mars 1514), Librairie Droz, Genève, Travaux d'Humanisme et Renaissance, $N^{\circ}$ DLXXII, 2017, $725 \mathrm{p}$.

1 Pendant vingt-cinq ans Jacques Santrot, en tant que directeur du Musée Dobrée, a été responsable de la conservation du reliquaire qui avait autrefois contenu le cœur d'Anne, duchesse de Bretagne et deux fois reine de France. C'est le plus célèbre objet précieux qui nous rappelle de manière tangible l'union politique du duché de Bretagne, auparavant largement indépendant, à la couronne de France à la fin du Moyen Âge et au début de la Renaissance. Comme J. Santrot l'écrit dans son avant-propos : «À tort ou à raison, le public breton considère ce fragile monument comme le symbole d'une relation mystique entre sa duchesse souveraine et reine, son peuple et son territoire ». Il attire aussi l'attention sur l'observation d'Alain Croix et de Didier Guivarc'h dans le catalogue qui accompagnait la belle exposition Anne de Bretagne, une histoire, un mythe (Château des ducs de Bretagne, Nantes, 2007), «Par le cœur de la reine déposé en l'église des Carmes, c'est un peu, entre mythe et réalité, le cœur même de la Bretagne qui a continué, au travers des siècles, à battre dans la cité ligérienne ». En 2014 une exposition qui connut un grand succès du reliquaire au château de Châteaubriant était accompagnée d'un autre excellent catalogue qui reprenait des thèmes similaires (Le cœur d'Anne de Bretagne, Milan, 2014).

2 La déposition solennelle du cœur d'Anne forme l'un des éléments majeurs de cet impressionnant volume lourd de plus de 700 pages; ce sont les secondes funérailles du 
titre, les premières étant celles de son corps à Saint-Denis. Cet ouvrage est le résultat remarquable de recherches assidues menées pendant de longues années par l'auteur sur tous les aspects des cérémonies entourant l'enterrement de la reine au début de 1514. Le sujet n'est pas nouveau, évidemment. Mais aucunes funérailles royales en France ne nous ont laissé auparavant des sources documentaires aussi riches qui permettent de retracer en détail ce qui se passa entre la mort et la sépulture, et peu de souverains depuis Anne ont été aussi consciemment exploités au moment de leur mort pour relayer des messages sur la nature du pouvoir royal et de son autorité.

Comme nous l'ont rappelé d'autres publications récentes, en particulier celles liées au cinq centième anniversaire de la mort d'Anne, ce triste événement fut célébré par un déferlement sans précédent de toutes sortes d'éloges funèbres, travaux manuscrits et imprimés qui ont attiré depuis l'attention d'antiquaires, chercheurs en littérature et historiens. Depuis le xix e siècle, la plupart de ces textes ont été édités et réédités, souvent plusieurs fois et de manière plus ou moins satisfaisante. Ici encore, l'anniversaire de 2014 a été un puissant stimulus pour de nouvelles éditions savantes de textes déjà connus ainsi que pour la publication de documents littéraires ou d'archives jusqu'alors méconnus ou même inédits qui ont ajouté encore plus de détails à ce que l'on savait déjà du cérémonial qui avait entouré la sépulture du corps d'Anne à SaintDenis et de son cœur à Nantes (BRown, Elizabeth A. R., BRown, Cynthia J., DEUfFIC, JeanLuc, JONES, Michael, «Qu'il mecte ma povre ame en celeste lumiere ». Les funérailles d'une reine Anne de Bretagne (1514). Textes. Images et manuscrits [Pecia, volume 15, 2012], Turnhout, Brepols, 2013, p. 1-336 couvre évidemment en grande partie le même champ d'étude et comprend certains des mêmes textes). La seconde grande section du livre de Santrot (p. 283-471) continue la tradition en éditant deux des textes clés (voir cidessous). Qu'est-ce donc alors qui fait du récit de Santrot, illustré de 32 photos en noir et blanc et de 36 planches en couleur, un ouvrage aussi remarquable qui fait autorité ?

Dans sa première partie (p. 19-280), il retrace avec une incomparable richesse de détails émanant de documents contemporains chaque étape du processus, jour après jour, depuis la mort de la reine et son embaumement à Blois jusqu'à l'ensemble des processions portant son corps à Paris et son cœur à Nantes. Il s'appuie sur deux principaux récits : la Commemoracion et advertissement de la mort de Madame Anne par Pierre Choque, héraut de Bretagne (dont on connait maintenant environ 40 copies manuscrites presque contemporaines, listées et classées ici avec soin, p. 286-304, avec une nouvelle édition du texte, p. 323-390, cf. BROWN, Elizabeth et al., Les funérailles d'une reine. Anne de Bretagne,1514, p. 7-190) et Le Trépas de l'Hermine regrettée dont seulement deux copies sont connues (Texte édité dans BROWN, Elizabeth et al., Les funérailles d'une reine. Anne de Bretagne (1514), p. 191-249), ainsi que sur les documents financiers fournis par les comptes de Guillaume de Beaune, trésorier et receveur général de la reine (Arch. dép. de Loire-Atlantique, E 208 no 2, texte complet édité ici pour la première fois p. 404-440, avec une analyse statistique p. 475-486). Ces trois documents fournissent la matière d'une narration détaillée que des recherches ultérieures sont peu susceptibles de modifier de manière substantielle. Parmi beaucoup d'autres choses, J. Santrot attache toujours une attention particulière au personnel de la maison de la reine et à l'entourage qui accompagna sa dépouille et il identifie autant de participants qu'il le peut. Le récit de Beaune est ici encore capital, mais J. Santrot exploite aussi efficacement un Estat des officiers qui ont accompaigné le corps de la feue royne... trouvé parmi les papiers saisis après la mort de Florimond Ier Robertet, trésorier de France et 
secrétaire des finances (Arch. nat., J 964 no 65, édité p. 391-403). Une grande partie de cette documentation prosopographique est regroupée dans l'annexe 5 « Qui est qui ? Le petit monde d'Anne de Bretagne» (p.509-601), pour une liste complémentaire, voir BRown, Elizabeth et al., Les funérailles d'une reine. Anne de Bretagne (1514), p. 289-321.). Mais en plus des personnages nommés qui prirent part aux différentes étapes des doubles funérailles d'Anne, J. Santrot a aussi essayé de calculer le nombre total de tous les autres qui y furent impliqués. Les résultats sont souvent surprenants: 1700 "pleurants » qui accompagnaient son corps à son départ du château de Blois pour la collégiale Saint-Sauveur (p.73-77), 12000 ou 13000 ou même plus dans le cortège quand le corps quitta finalement la ville (p. 89), un cortège d'une importance similaire à Paris (p. 147-148), peut-être 3000 pour accompagner son cœur à Nantes (p. 240), 4800 pauvres qui reçurent des aumônes à la fois à Notre-Dame de Paris et à Saint-Denis (p.170). De tels chiffres impliquent des problèmes d'organisation que même une abondance de sources ne parvient pas à éclairer de façon satisfaisante (Comment ceux qui prirent part à ces processions savaient où et quand se rassembler? Comment étaient-ils tous nourris et logés pour la nuit? Comment les pauvres furent-ils choisis et canalisés ?). La capacité de la couronne et de ses officiers à organiser un événement et un cérémonial aussi complexe et prolongé sur plus de deux mois est remarquable, même si les rouages nous en sont largement cachés. J. Santrot est aussi capable de calculer avec une remarquable précision le nombre de messes dites ou chantées à chaque étape, la quantité ahurissante de cire consumée par les chandelles et les torches, le nombre impressionnant d'aunes de tissu de deuil et de paires de chaussures achetées et distribuées, et même le nombre de carillonnements et enfin l'énorme coût total (60 000 livres au moins, p. 184-188, même sans les dépenses de l'inhumation du cœur d'Anne à Nantes) dont la plus grande partie fut supportée par Louis XII lui-même, certainement en témoignage de ses sentiments sincères envers son épouse, mais aussi comme une démonstration délibérée de la magnificence royale.

5 J. Santrot établit fréquemment des parallèles ou montre les contrastes avec les funérailles d'autres personnages importants du Moyen Âge et de la Renaissance, grâce à la fois aux récits d'antiquaires et à de nombreuses études modernes actuellement en plein essor et dont la liste est donnée dans l'abondante bibliographie, p. 625-656. Dans un travail qui est déjà une telle mine d'informations, il fournit une étude des vicissitudes connues par la suite par le célèbre cœur reliquaire : son ouverture après une inondation en 1590 (où le cœur de la reine, selon un récit postérieur " était entier, mais encore tout brillant d'un sang vermeil »), sa restauration au début du xvirle siècle, sa préservation presque miraculeuse à la Révolution, son séjour à Paris entre 1793 et 1819 , son retour à Nantes et ses pérégrinations ultérieures ainsi que des controverses au sujet de son lieu de conservation. Il récapitule aussi les récentes investigations scientifiques pendant les procédures de conservation avec des descriptions détaillées de son état matériel et des inscriptions qui y figurent (p. 196-221, 497-507). Un glossaire et deux index très complets (un «Index des noms de personnes, fonctions, titres, institutions, ordres religieux» (p.657-701) et un "Index des noms de lieux, monuments, établissements religieux » (p. 703-716), complètent le volume.

6 Compte tenu de la taille et de la complexité de cet ouvrage, il y a inévitablement quelques petites imperfections, mais ce sont en général des erreurs mineures, quoique quelques dates posent problème. L'embaumement des personnes royales commence avec Louis VIII en 1226 et Charles V meurt en 1380 (p. 31) ; le pape Martin V vivait sous le règne du duc Jean $\mathrm{V}$ (p. 132); la duchesse-reine eut son « entrée joyeuse » à Tours en 
1497 (p. 189); son cœur fut inhumé en 1514, non 1498 (p. 195); la reine Marie d'Angleterre entra dans Paris en 1514, et non en 2014 (p. 278) ; le premier traité de Guérande date de 1365 (p. 519) ; Jean IV épousa Marie, fille d'Édouard III d'Angleterre en 1361 , et elle mourut quelques mois plus tard et non en 1355 , erreur induite à la suite de La Borderie (p.526). Un ou deux renvois à des planches d'illustrations semblent erronés. Par ailleurs, il y a très peu de coquilles dans un livre dont la belle maquette et la présentation agréable sont à mettre au crédit d'une célèbre maison d'édition qui a bien servi son auteur dans ce qui a été une œuvre de grande dévotion à la mémoire de la duchesse Anne. 